\title{
Questes
}

\section{Les hommes illustres : éléments bibliographiques}

\section{Marion Chaigne-Legouy et Anne Salamon}

\section{(2) OpenEdition}

\section{Journals}

\section{Édition électronique}

URL : http://journals.openedition.org/questes/1653

DOI : 10.4000/questes. 1653

ISSN : 2109-9472

\section{Éditeur}

Les Amis de Questes

\section{Édition imprimée}

Date de publication : 15 octobre 2009

Pagination : 124-128

ISSN : 2102-7188

\section{Référence électronique}

Marion Chaigne-Legouy et Anne Salamon, «Les hommes illustres : éléments bibliographiques », Questes [En ligne], 17 | 2009, mis en ligne le 01 janvier 2014, consulté le 15 septembre 2020. URL: http://journals.openedition.org/questes/1653 


\section{Éléments bibliographiques ${ }^{1}$}

\section{Les Hommes illustres : réception et relecture d'un genre antique}

AlAZARD, Jean, «Sur les Hommes Illustres », in Il mondo antico nel Rinascimento : Atti del $V$ convegno internazionale di studi sul Rinascimento (Firenze, Palazzo Strozzi, 2-6 settembre 1956), Florence, Sansoni, 1958, p. 275-277.

Bozzolo, Carla, «La lecture des classiques par un humaniste français: Laurent de Premierfait », in Dario CECChETTI, Lionello Sozzi, Louis TERREAUX (éd.), L'Aube de la Renaissance, Genève, Slatkine (Bibliothèque Franco Simone, 18), 1991, p. 67-81.

CACIORgna Marilena, Guerrini, Roberto, La Virtù figurata. Eroi ed eroine dell'antichità nell'arte senese tra Medioevo e Rinascimento, Sienne, Protagon Editori, 2003.

Dolbeau, François, «Une refonte wisigothique du De viris illustribus d'Isidore », in Louis Holtz et Jean-Claude Fredouille (éd.), De Tertullien aux Mozarabes. Mélanges offerts à Jacques Fontaine, t. II : Antiquité tardive et Christianisme ancien $\left(\mathrm{VI}^{\mathrm{e}}-\mathrm{IX}^{\mathrm{e}}\right.$ siècle), Paris, Institut d'Études Augustiniennes, 1992, p. 41-56.

DonATO, Maria Monica, "Gli eroi romani tra storia ed "exemplum". I primi cicli umanistici di Uomini Famosi », in Salvatore SETTis (éd.), Memoria dell'antico nell'arte italiana, vol. II : I generi e i temi ritrovati, Turin, Giulio Einaudi editore, 1985, p. 95152.

JEUDY, Colette, «Biographies d'hommes illustres dans la bibliothèque de Guillaume Fillastre ", in Didier MARCOTTE (éd.), Humanisme et culture géographique à l'époque du Concile de Constance: autour de Guillaume de Fillastre, Actes du Colloque de l'Université de Reims (18-19 novembre 1999), Turnhout, Brepols, 2002, p. 77-93.

Joost-Gaugier, Christiane L., « The Early Beginnings of the Notion of Uomini Famosi and the De Uiris Illustribus in Greco-Roman Literary Tradition », Artibus et Historiae, 6 (1982), p. 97-115.

KoHL, Benjamin G., "Petrarch's prefaces to De Viris Illustribus », History and Theory, 13/2 (1974), p. 132-144.

MoISAN, André, «Héroïsme et sainteté. Interférences entre deux types de l'idéal au Moyen Âge », in Jean-Charles PAYEN (éd.), La Légende arthurienne et la Normandie (hommage à René Bansard), Condé-sur-Noireau, Charles Corlet, 1983, p. 45-71.

Mommsen, Theodor E., "Petrarch's conception of the "Dark Ages", especially in De viris illustribus », in Paula FINDLEN (éd.), The Italian Renaissance: The Essential

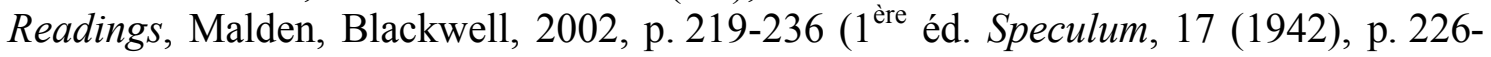
242).

SchmitT, Annegrit, «Zur Wiederbelegung der Antike im Trecento », Mitteilungen des Kunsthistorisches Instituts in Florenz, XVIII (1974), p. 167-218.

\footnotetext{
${ }^{1}$ Nous remercions Jean-Baptiste Delzant pour les références relatives à l'histoire de l'art qu'il nous a gentiment communiquées.
} 
Young, Frances M., "Classical genres in Christian guise; Christian genres in classical guise ", in Frances M. Young, Lewis Ayres, Andrew Louth (éd.), The Cambridge History of Early Christian Literature, Cambridge University Press, 2004.

\section{Les héros antiques revisités au Moyen Âge}

Aerts, Willem Jonan, Gosman, Martin (éd.), Exemplum et Similitudo. Alexander the Great and other heroes as points of reference in medieval literature, Groningue, Egbert Forsten, 1988.

Buchthal, Hugo, Historia Troiana. Studies in the History of Medieval Secular Illustration, London, Warburg institute, University of London; Leiden, E. J. Brill, 1971.

Meniel, Bruno, et RiBemont Bernard (dir.), La Figure de Jules César au Moyen Âge et à la Renaissance, Cahiers de recherches médiévales, n¹3 et 14 (numéros spéciaux, 2006 et 2007).

\section{Sur les recueils de vies d'Hommes illustres : une approche littéraire}

Aurigemma, Marcello, «Petrarca e la storia : osservazioni sulle biografie del De viris illustribus de Adamo ad Ercole», in Scrittura e società. Studi in onore di Gaetano Mariania, Rome, Herder, 1985, p. 53-74.

Bozzolo, Carla, Manuscrits des traductions françaises d'œuvres de Boccace: XV siècle, Padou, Editrice Antenore (Medioevo e umanesimo 15), 1973.

BROWN-GRANT, Rosalind, « Des hommes et des femmes illustres : modalités narratives et transformations génériques chez Pétrarque, Boccace et Christine de Pizan ", in Liliane DulaC et Bernard RiBEmont (éd.), Une femme de lettres au Moyen Âge: Études autour de Christine de Pizan, Orléans, Paradigme (Medievalia, 16), 1995, p. $469-480$.

Cerquiglini-Toulet, Jacqueline, «À la recherche des pères: la liste des auteurs illustres à la fin du Moyen Âge », MLN, vol. 116, n4 (sept. 2001), p. 630-643.

—, «Fama et les Preux : nom et renom à la fin du Moyen Âge », Médiévales, 24 (printemps 1993), p. 35-44.

Codoñer, Carmen, «El libro de Viris illustribus de Ildefonso de Toledo », La Patrología toledano-visigoda, XXVII Semana Española-Català de Teología (25-29 septembre 1967, Tolède), Madrid, Inst. Francisco Suárez, 1970, p. 337-348.

EICHEL-LoJkine, Patricia, Le Siècle des Grands Hommes. Les recueils de Vies d'hommes illustres avec portraits du XVI siècle, Louvain, Peeters, 2001.

FILOSA, Elsa, «Petrarca, Boccaccio e le mulieres clarae: dalla Familiare XXI 8 al De mulieribus claris », Annali d'Italianistica, 22 (2004), p. 381-95. 
GATHerCole, Patricia M., « The French translations of Boccaccio », Italica, 45 (1969), p. 123-209.

Hauvette, Henri, «Les plus anciennes traductions françaises de Boccace », in Études sur Boccace, Torino, Bottega d'Erasmo, 1968, p. 151-294.

Jeanroy, Alfred, « Boccace et Christine de Pisan. Le De claris mulieribus, principale source du Livre de la Cité des dames », Romania, 48 (1922), p. 93-105.

MCLeod, Glenda, Virtue and Venom. Catalogs of Women from Antiquity to the Renaissance, Ann Arbor, The University of Michigan Press, 1991.

Mombello, Gianni, «Per la fortuna del Boccaccio in Francia », Studi sul Boccaccio, 1 (1963), p. 415-444.

Morini, Agnès (éd.), Figure, figures. Portraits de femmes et d'hommes célèbres, ou moins, dans la littérature italienne (actes du colloque du CERCLI, Saint-Etienne, mai 2000), Publications de l’Université de Saint-Etienne, 2002.

Petitmengin, Pierre, «Le De viris illustribus de Sigebert de Gembloux. À propos d'une édition récente ", Le Moyen Âge. Revue d'histoire et de philologie, 85/2 (1979), p. 317-322.

Rouse, Mary A., et Rouse, Richard H., « Bibliography before print : the medieval De viris illustribus", in Peter GANZ (éd.), The Role of the Book in Medieval Culture. Proceedings of the Oxford International Symposium 29 septembre-1 ${ }^{\text {er }}$ octobre 1982, Bibliologia. Elementa ad Librorum Studia Pertinentia, Turnouht, Brepols, 1986, p. 133153.

SCHRÖDER, Horst, Der Topos der Nine Worthies in Literatur und bildender Kunst, Göttingen, 1971.

TESNIERE, Marie-Hélène, "La réception des Cas des nobles hommes et femmes de Boccace en France au $\mathrm{XV}^{\mathrm{e}}$ siècle, d'après l'illustration des manuscrits », in Michelangelo PiCone (éd.), Autori e lettori di Boccaccio. Atti del Convegno internazionale di Certaldo (20-22 settembre 2001), Firenze, Franco Cesati Editore, 2002, p. 387-402.

\section{Fresques et cycles iconographiques autour des Hommes illustres}

ARASSE, Daniel, «Portrait, mémoire familiale et liturgie dynastique : Valerano-Hector au château de Manta », in Augusto Gentili (éd.), Il ritratto e la memoria, Rome, Bulzoni, 1989, p. 93-112 et plus particulièrement p. 102-103.

Benazzi, Giordana, Mancini, Franscesco Federico (éd.), Il palazzo Trinci di Foligno, Pérouse, Quattroemme, 2001. 
CAglioti, Francesco, «Francesco Sforza e il Filelfo, Bonifacio Bembo e "compagni" : nove prosopopee inedite per il ciclo di antichi eroi e eroine nella Corte Ducale dell'Arengo a Milano (1456-1461 circa)», Mitteilungen des Kunsthistorischen Institutes in Florenz, XXXVIII (1994), p. 183-217.

CANFIELD, Gabriella Befani, «The reception of Flemish art in Renaissance Florence and Naples », in Maryan W. Ainsworth (éd.), Petrus Christus in Renaissance Bruges : an Interdisciplinary Approach, Turnhout, Brepols, 1995, p. 35-42.

Dal Pogetto, Paolo, «Ciò che era finora invisibile nella camera picta del Palazzo Ducale a Urbino », Bollettino d'arte, s. VI, LXXVII, 65 (1991), p. 71-78.

D’Ancona, Paolo, « Gli affreschi del Castello di Manta nel Saluzzese », L'Arte, VIII/ I (1905), p. 94-106 et p. 184-198.

DunN, Josephine M., «Andrea del Castagno e i Carducci : documenti vecchi e nuovi riguardanti la villa Carducci in Firenze », Archivio storico italiano, 147/540 (1989), p. $251-275$.

GALASSI, Cristina, " La decorazione pittorica di Palazzo Trinci di Foligno. I. Cronologia e stile », Annali della Facoltà di Lettere e Filosofia dell'Università degli Studi di Perugia, 26, n. s. 12, 2, (1988-1989), Studi Storico Antropologici, p. 177-214.

GiLbert, Creighton E., "The fresco by Giotto in Milan », Arte Lombarda, 47-48 (1977), p. 31-72.

GUERRINI, Roberto, « Dal testo all'immagine. La "pittura di storia" nel Rinascimento », in Salvatore SETTIS (éd.), Memoria dell'antico nell'arte italiana II : I generi e i temi ritrovati, Turin, 1985, p. 44-93.

—, «Effigies procerum. Modelli antichi (Vergilio, Floro, De viris illustribus) negli epigrammi del Salutati per Malazzo Vecchio a Firenze », Athenaeum : Studi periodici di letteratura e storia dell'antichità, 71/1 (1993), p. 210-12.

—, « Dai cicli di uomini famosi alla biografia dipinta. Traduzioni latine delle Vite di Plutarco ed iconografia degli eroi nella pittura murale del Rinascimento », Fontes 1-2 (1998), p. 137-158.

Joost-Gaugier, Christiane L., «A Rediscovered Series of Uomini Famosi from Quattrocento Venice », The Art Bulletin, 58 (1976), p. 184-195.

- " Giotto's Hero Cycle in Naples: a Prototype of Donne Illustri and a Possible Literary Connection », Zeitschrift für Kunstgeschichte, 43 (1980), p. 311-318.

Marcelli, Fabio, «Uomini illustri dell'antichità », in Andrea De Marchi (éd.), Pittori a Camerino nel Quattrocento, Jesi, Banca delle Marche, 2002, p. 257-263.

Mommsen, Theodor E., « Petrarch and the Decoration of the Sala Virorum Illustrium in Padua », The Art Bulletin, 34/2 (1952), p. 95-116. 
Morpurgo, Salomone, Un affresco perduto di Giotto nel Palazzo del Podestà di Firenze, Florence, Carnesecchi, 1897.

RESSORT, Claudie, et REYNAUd, Nicole, « Les portraits d'hommes illustres au Studiolo d'Urbino au Louvre par Juste de Gand et Pedro Berruguete ", Revue du Louvre et des Musées de France, 41/1 (1991), p. 82-116.

Salmi, Mario, « Romani Illustri fra Gotico e Rinascimento », Colloqui del Sodalizio, 4 (1973-1974), p. 135-138.

—, «I Trionfi e il De Viris Illustribus nell'Arte del Primo Rinascimento », in Convegno internazionale Francesco Petraca (Roma - Arezzo - Padova - Arquà Petrarca, 24-27 aprile 1974), Atti dei Convegni Lincei X, Rome, 1976, p. 23-47.

SCHAEFFER, Emil, «Über Andrea del Castagno’s uomini famosi», Repertorium für Kunstwissenschaft, 25 (1902), p. 170-177.

TeZA, Laura, Fra ei poggi e l'aqque al laco Transimeno. Pietro Vannucci, Maturanzio e gli Uomini Famosi nella Perugia dei Baglioni, Pérouse, Quattroemme, 2008.

VAdÉE, Claire, «Gli affreschi di Palazzo Trinci e la pittura folignate tra Trecento e Quattrocento ", in Signorie in Umbria tra Medioevo e Rinascimento : l'esperienza dei Trinci, Congresso storico internazionale, Foligno, 10-13 décembre 1986, Pérouse, Deputazione di Storia Patria per l'Umbria, 1989, p. 403-427.

\section{Hommes illustres et programme politique}

ARASSE, Daniel, « Frédéric dans son cabinet : le studiolo d'Urbino », in Le Sujet dans le tableau. Essais d'iconographie analytique, Paris, Flammarion, 1997 (nouvelle édition 2008), p. 17-30.

DieTL, Albert, «Der Triumph des Ottone Visconti. Zu Datierung und Programm des Freskenzyklus in der Rocca von Angera », Sitzungsberichte der Kunstgeschichtlichen Gesellschaft in Berlin, 41/42 (1994, parution 1998), p. 41-44.

Galassi, Cristina, «La Sala dei Giganti di Palazzo Trinci : Iconologia di un ciclo "politico" ", Bollettino Storico della Città di Foligno, 14 (1990), p. 155-182.

LEROY, Béatrice, Histoire et politique en Castille au XV siècle. Les hommes illustres de Castille de Hernando del Pugar, Limoges, PULIM, 2001.

—, «La mémoire politique de la Castille au $\mathrm{XV}^{\mathrm{e}}$ siècle: une galerie des hommes illustres », in Jean KERHERVE et Albert Rigaudiere (éd.), Finances, pouvoirs et mémoires : mélanges offerts à Jean Favier, Brest, Fayard, 1999, p. 301-312.

PuCcioni, Giulio, La fortuna medievale della "Origo gentis romanae », Florence, Messine, G. d'Anna, 1958. 